\title{
Urinary tract obstruction in the mouse: the kinetics of distal nephron injury
}

\author{
Michael J Hiatt ${ }^{1,2}$, Larissa Ivanova ${ }^{3}$, Peter Trnka ${ }^{4}$, Marc Solomon ${ }^{1}$ and Douglas G Matsell ${ }^{1,5}$
}

Congenital urinary tract obstruction is the single most important cause of childhood chronic kidney disease. We have previously demonstrated that human and primate fetal obstruction impairs the development, differentiation, and maturation of the kidney. Research using postnatal rodent models has primarily focused upon the role of proximal tubular injury, with few reports of collecting duct system pathology or the suitability of the postnatal models for examining injury to the distal nephron. We have employed the mouse unilateral ureteric obstruction (UUO) model and examined time points ranging from 1 to 14 days of obstruction. Many of the key features of fetal collecting duct injury are replicated in the postnatal mouse model of obstruction. Obstruction causes a sixfold increase in myofibroblast accumulation, two- to threefold dilatation of tubules of the distal nephron, $65 \%$ reduction of principal cell aquaporin 2 expression, $75 \%$ reduction of collecting duct intercalated cell abundance, and disruption of E-cadherin- and $\beta$ cateninmediated collecting duct epithelial adhesion. Notably, these features are shared by the distal and connecting tubules. This work confirms that distal nephron pathology is a significant component of postnatal mouse UUO. We have highlighted the utility of this model for investigating collecting duct and distal tubule injury and for identifying the underlying mechanisms of the distal nephron's contribution to the repair and fibrosis.

Laboratory Investigation (2013) 93, 1012-1023; doi:10.1038/labinvest.2013.90; published online 5 August 2013

KEYWORDS: collecting duct; distal tubule; obstruction; unilateral ureteric obstruction

Congenital urinary tract obstruction is the single most important cause of chronic kidney disease in children. In the fetus, obstruction of urinary flow during the critical stages of renal nephrogenesis alters branching morphogenesis, decreases nephron endowment, and disrupts normal tubulointerstitial development. ${ }^{1-4}$ We have previously demonstrated that fetal obstruction in the human and primate kidney causes substantial medullary injury, including interstitial expansion, peritubular $\alpha$ smooth muscle actin ( $\alpha \mathrm{SMA})$ collar formation, and collecting duct epithelial dysfunction. ${ }^{1-3}$ Additional work is required to further identify how medullary and collecting duct injury contributes to and modulates the pathophysiology and progression of injury following obstruction.

The rodent unilateral ureteric obstruction (UUO) models are the best-described models of urinary tract obstruction ${ }^{5-16}$ and are widely used due to their ease of manipulation and the availability of transgenic animals. These models are predominantly postnatal models with much of the work focused on the role of the proximal convoluted tubule and on late time points in which the interstitial and fibrotic responses predominate. ${ }^{17-20}$ Although early changes in tubular hydrodynamics, tubular dilatation, and medullary injury have been briefly described following obstruction, our knowledge of the contributions of these factors and the potential cellular responses they invoke is lacking.

We have previously demonstrated that the distal nephron, including the collecting duct, has an important role in the pathogenesis of obstruction in the fetal human and primate kidney. ${ }^{1-3}$ Here, we demonstrate that despite the postnatal nature of the mouse UUO model, this model replicates many of the key pathogenic features of fetal obstruction, making it a suitable and powerful model for investigating early medullary and distal nephron injury.

\footnotetext{
${ }^{1}$ Department of Pediatrics, Child and Family Research Institute, University of British Columbia, Vancouver, BC, Canada; ${ }^{2}$ Developmental Biology and Regenerative Medicine Research Program, Saban Research Institute, Children's Hospital of Los Angeles, Los Angeles, CA, USA; ${ }^{3}$ Jack Bell Research Centre, University of British Columbia, Vancouver, BC, Canada; ${ }^{4}$ Department of Pediatrics and Child Health, University of Queensland, Queensland Child and Adolescent Renal Service, Brisbane, QLD, Australia and ${ }^{5}$ Division of Nephrology, British Columbia Children's Hospital, Vancouver, BC, Canada Correspondence: Dr Douglas G Matsell, MDCM, Division of Nephrology, British Columbia Children's Hospital, 4480 Oak Street, Vancouver V6H 3V4, BC, Canada. E-mail: dmatsell@cw.bc.ca
} 


\section{MATERIALS AND METHODS} Animals and Surgical UUO

All animal protocols were approved by the Committee on Animal Care at the University of British Columbia. Male mice aged 6 to 8 weeks (Jackson Labs, Bar Harbor, ME, USA) were used in all experiments. For induction of surgical obstruction, mice were anesthetized with $2-3 \%$ isoflurane and administered pre-operative medications and isotonic saline. Following a flank incision, the right ureter was isolated and either ligated with a 5-0 prolene suture or sham manipulated. Following closure, mice were monitored until fully recovered. For all analyses, obstructed and sham-manipulated kidneys were compared for a minimum of three mice per time point $(N=3)$.

\section{Sample Collection and Processing}

Mice were euthanized at 1,2,3,6, 9, or 14 days post-surgery and sham or obstructed kidneys were collected and immediately fixed for embedding or were flash frozen. For paraffin-embedding, kidneys were immediately fixed in $4 \%$ paraformaldehyde overnight, dehydrated through graded ethanols, perfused in a 1:1 mix of toluene and paraffin wax before sectioning at $6 \mu \mathrm{m}$ thickness and mounting on Superfrost Plus slides (Fisher Scientific, Ottawa, Canada). Formalin-fixed paraffin-embedded tissue sections were deparaffinized in xylene and rehydrated by passage through graded ethanols. Following a PBS wash, samples were either stained with hematoxylin and eosin, or processed for fluorescent immunohistochemistry as below.

\section{Fluorescent Immunohistochemistry}

Formalin-fixed paraffin-embedded tissues were subjected to $40 \mathrm{~min}$ of heat-induced epitope retrieval in $10 \mathrm{mM}$ citrate buffer ( $\mathrm{pH} \mathrm{6)}$ in a domestic food steamer. Following a PBS wash, tissue sections were blocked for $1 \mathrm{~h}$ in buffer containing $0.1 \mathrm{M}$ PBS solution containing $1 \%$ bovine serum albumin (BSA), $0.1 \%$ cold fish-skin gelatin, $0.1 \%$ Triton X-100, $0.05 \%$ Tween-20, $0.5 \%$ sodium azide and $2 \%$ of either goat or horse normal serum. Primary antibodies were diluted in $0.1 \mathrm{M}$ PBS containing $1 \%$ BSA, $0.1 \%$ cold fish-skin gelatin and $0.5 \%$ sodium azide and incubated overnight at $4{ }^{\circ} \mathrm{C}$. Next, sections were incubated for $1 \mathrm{~h}$ with AlexaFluorconjugated secondary antibodies (Life Technologies, Burlington, Canada) diluted in 0.1 M PBS containing 0.5\% sodium azide. Slides were mounted with Prolong Gold mounting media with antifade or, for nuclear staining, in Prolong Gold + DAPI mounting media with antifade (Life Technologies).

\section{Microscopy}

Epifluorescent microscopy was performed on a Leica DM4000B upright microscope. Images were captured with a Retiga 1300i camera with RGB filter wheel (QImaging, Surrey, Canada) and were processed and merged using Openlab software (Perkin Elmer, Waltham, MA, USA).
Confocal analysis was performed on a Leica SP5 II Confocal microscope, using Leica's LAS AF software for image acquisition. Post-capture processing was performed using ImagePro 6.2 software (Media Cybernetics, Rockville, MD, USA). Tiled images of each kidney sections for Figure 4 were captured using a DX61 microscope (Olympus, Center Valley, PA, USA) and processed with ImagePro 6.2 (Media Cybernetics). Standardized image acquisition and post-capture processing settings were used to capture images for each figure and different magnification.

\section{Morphometric Analysis}

For quantification of interstitial $\alpha$ SMA-positive cell infiltration or expansion, the area of positively staining cells was measured in sections stained for $\alpha \mathrm{SMA}$ via fluorescent immunohistochemistry as above. Ten representative $40 \times$ high-power fields were captured per kidney from both cortex and outer medulla with equal representation of the inner and outer portions of each region. Using a 99-point grid (11 vertical and 9 horizontal lines), points that fell on $\alpha$ SMA-positive area were counted from three kidneys for each time point for each experimental condition (sham, obstructed). Points lying on large vessels of the vasculature were not counted.

To compare tubular dilatation in obstructed vs sham kidneys, luminal diameters (cell apex to cell apex) were measured in medullary aquaporin 2 (AQP2)-expressing collecting ducts and in cortical V-type proton ATPase (vATPase)-expressing distal convoluted and connecting tubule segments using ten representative $40 \times$ high-power medullary and cortical fields per kidney. In obstructed kidneys, dilatation of the cortical collecting duct was less severe than in either the medullary collecting duct or distal convoluted and connecting tubules, and was therefore not measured. Outer medullary fields were captured from both the inner and outer stripe as for $\alpha \mathrm{SMA}$ morphometric analysis. Representative cortical fields were captured from regions of the cortical labyrinth lying between the medullary rays. Absolute measurements were made on captured images using a measuring tool in the OpenLab software (Improvision) calibrated to a micrometer.

Intercalated cell numbers were manually counted as described previously. ${ }^{1}$ Briefly, vATPase-positive cells were counted in collecting ducts of 3- and 14-day sham and obstructed kidneys and expressed as a percentage of the total number of cells per duct as determined by counting of DAPI-stained nuclei.

\section{Analysis of Apoptosis}

Terminal deoxynucleotidyl transferase dUTP nick end labeling (TUNEL) analysis was performed using the ApoptagFITC kit (EMD Millipore, Billerica, MA, USA). Formalinfixed paraffin-embedded tissue was deparaffinized and treated with $20 \mu \mathrm{g} / \mathrm{ml}$ of Proteinase K (Sigma, St Louis, MO, USA) as directed. Following incubation with terminal transferase, sections were incubated with the provided 
anti-digoxigenin FITC-conjugated secondary antibody. All slides were counterstained with rabbit anti-AQP2 (1:100, Sigma) and an Alexafluor-568-conjugated secondary antibody.

\section{Immunoblotting}

Protein lysates for immunoblotting were made from $150 \mu \mathrm{m}$ trim sections from flash frozen kidneys collected in protein lysis buffer containing 50:50 TPER Buffer (Fisher Scientific)/ SDS buffer containing protease inhibitors (Roche, Laval, Canada). Protein lysates were quantified using a NanoDrop spectrophotometer (Fisher Scientific). Normalized protein lysates were mixed with $5 \times$ sample buffer and electrophoresed on a $10 \%$ gel using SDS-PAGE. Following transfer to PVDF membrane (Bio-Rad, Mississauga, Canada), and blocking with $5 \%$ BSA, blots were incubated with primary antibodies in TBST containing 5\% BSA for $1 \mathrm{~h}$ at room temperature or at $4{ }^{\circ} \mathrm{C}$ overnight. Blots were incubated for $1 \mathrm{~h}$ at room temperature with horseradish peroxidase-conjugated anti-rabbit or -mouse secondary antibodies (Cell Signaling, Danvers, MA, USA), treated with ECL solution and exposed on radiography film. Developed films were scanned on a commercial desktop scanner and analyzed via Image J software (NIH, Bethesda, MA, USA).

\section{Antibodies}

Primary antibodies used: (1) immunohistochemistrymouse anti- $\alpha$ SMA (Sigma), rabbit anti-type IV collagen (Coll IV, Fitzgerald Industries, Acton, MA, USA), rabbit antiAQP2 (Sigma), rabbit anti-vATPase (Santa Cruz, Santa Cruz, CA, USA), mouse anti-E-cadherin (ECad) (BD Bioscience, Mississauga, Canada); rabbit anti- $\beta$ catenin ( $\beta$ Cat) (Cell Signaling); (2) immunoblotting—rabbit anti-AQP2 (Sigma), rabbit anti-vATPase (Santa Cruz). The secondary antibodies used: (1) immunohistochemistry—goat-derived AlexaFluor-488 and -568 conjugates against anti-mouse or anti-rabbit IgG heavy and light chains (Life Technologies); (2) immunoblotting-horseradish peroxidase-conjugated anti-mouse or anti-rabbit (Cell Signaling).

\section{Statistical Analysis}

For each experiment, a minimum of three animals was used $(N=3)$. For densitometry and intercalated cell counts, results are presented as the mean of the values for three animals and expressed with their standard deviations. For morphometric analysis of $\alpha \mathrm{SMA}$ area, results are expressed as the number of aSMA-positive points per high-power field and expressed with their standard deviations. For measurement of tubular dilatation, results are expressed as the mean luminal diameter per group and expressed with their standard deviations. Group comparison measurements were analyzed by two-way ANOVA and TUKEY post hoc testing using SPSS Statistics (IBM, Armonk, New York, USA). Significant findings were determined by $P \leq 0.5$.

\section{RESULTS}

\section{General Features of UUO}

Following surgical ligation, the mice were allowed to recover for $1,2,3,6$, 9, or 14 days. Successful ligation of the ureter was confirmed by the examination of the gross pathology of the kidney upon kill and tissue collection. As expected, obstruction of the ureter by surgical ligation resulted in the accumulation of urine and distension of the ureter proximal to the site of occlusion. Obstruction caused visible swelling and progressive hydronephrosis in the ligated kidneys. Examination of renal histology following obstruction revealed that the accumulation of urine and progressive distension of the ureter that extended through the renal pelvis to the urinary space of the unipapillary mouse kidney (Figure 1a) with progressive compression of the outer renal medulla, and to a lesser extent, of the renal cortex (Figures $1 \mathrm{~b}$ and c). Kidneys obstructed for 14 days exhibited severe hydronephrosis, tissue compression, and parenchymal injury.

\section{Interstitial Response}

Obstructive kidney injury is associated with dramatic changes to the interstitial compartment. ${ }^{21-24}$ Interstitial expansion and tubular epithelial remodeling are key features of the early response to obstructive injury and occur even before the onset of the interstitial fibrosis that is evident at later time points. Specifically, the accumulation of myofibroblasts expressing $\alpha \mathrm{SMA}$ is a hallmark feature of both tissue repair and fibrosis. In sham-operated kidneys at all time points, aSMA immunoreactivity was not observed in the tubular epithelium and was restricted to the vasculature, calyceal lining, and a small population of interstitial cells in the medulla and cortex (Figure 2). Following UUO, expansion of the interstitial space and accumulation of interstitial $\alpha$ SMApositive cells occurred rapidly in both the outer medulla and, to a lesser extent, in the cortex (Figure 2), and increased progressively with prolonged obstruction.

The magnitude and localization of interstitial $\alpha$ SMA within specific regions of the kidney were assessed using morphometric analysis. At all time points, the medullary $\alpha \mathrm{SMA}$ counts were higher than those of the cortex with a significant effect of both obstruction and time on the area of $\alpha \mathrm{SMA}$ immunoreactivity (Figures $2 \mathrm{~b}$ and $\mathrm{c}$ ). In both cortex and outer medulla, post hoc testing demonstrates no differences between any of the sham groups, but a significant increase $(P<0.05)$ in $\alpha$ SMA area after 2 days of obstruction before reaching a plateau from 6 to 9 days. In the outer medulla, $\alpha$ SMA area increased significantly $(P<0.05)$ from 2 to 6 days, with an intermediate increase in $\alpha \mathrm{SMA}$ area at 3 days obstruction. In the cortex, $\alpha$ SMA area does not differ between 2 and 3 days, but increased significantly $(P<0.05)$ from 3 to 6 days. These results demonstrate that $\alpha$ SMApositive cells rapidly accumulate in the renal interstitium following obstruction, and that this effect is more pronounced in the medulla than in the cortex. 
a

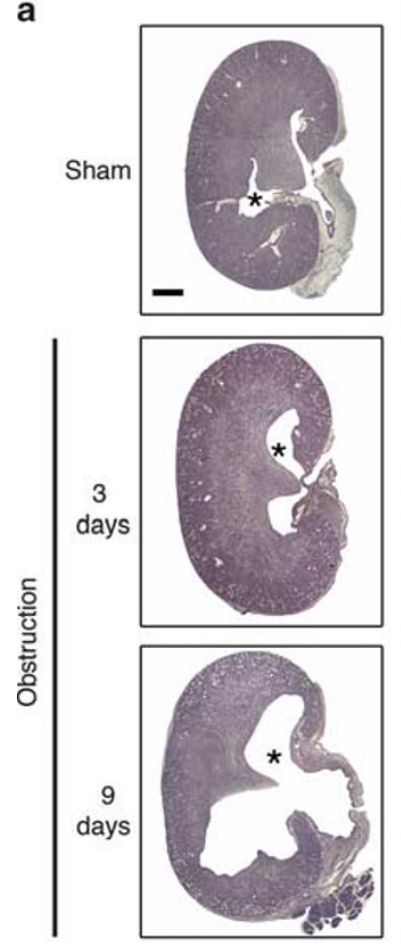

b
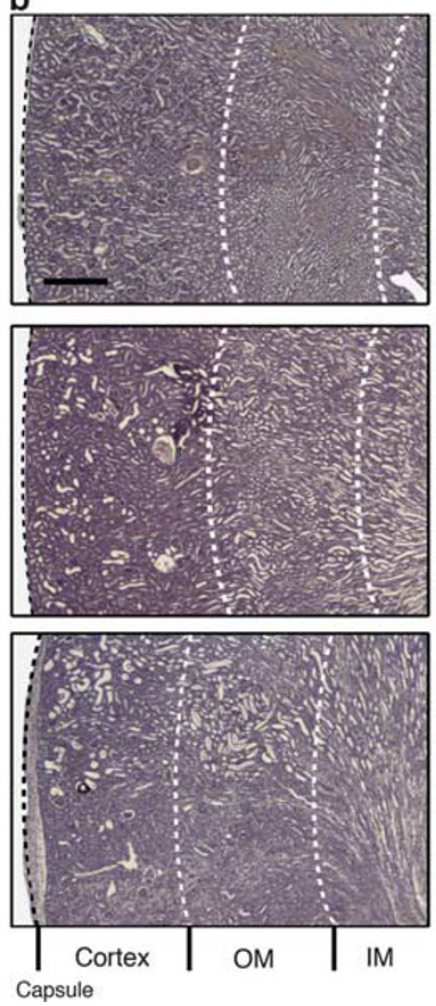

c
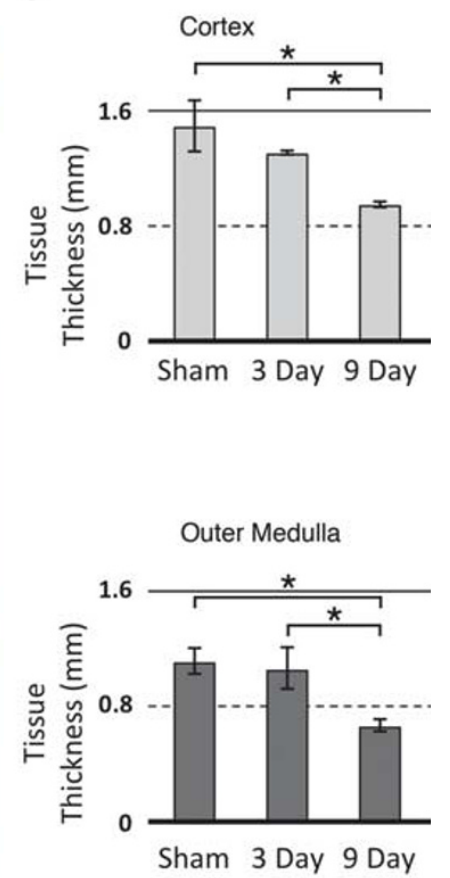

Figure 1 General features of UUO. Following urinary tract obstruction, the accumulation of urine proximal to the site of ligation causes (a) progressive distension of the ureter and calyx (asterisk), and (b) associated compression of the renal outer medulla (OM) and cortex. IM, inner medulla. (c) Mean cortical and medullary tissue thickness decreases with prolonged obstruction. ${ }^{*} P<0.05$. Error bars denote standard deviation. Scale bars: (a) $1 \mathrm{~mm}$, (b) $400 \mu \mathrm{m}$.

As with $\alpha \mathrm{SMA}$, reorganization and deposition of collagen are often key interstitial features following obstruction. The deposition and accumulation of collagens I and III are frequently used as markers of fibrosis at later time points following obstruction. ${ }^{25-28}$ Coll IV, on the other hand, is the major component of the basement membrane. Previous studies have demonstrated abnormal expression of Coll IV to be a marker of epithelial injury and tubular remodeling., ${ }^{2,3,29}$ In sham-operated kidneys, Coll IV is localized to the basement membranes of all tubules and ducts of the outer medulla and cortex and surrounding the vasculature (Figure 3). Obstruction results in the expansion of the cortical and medullary interstitial space and in the disruption and duplication of Coll IV basement membranes that surround the adjacent tubules or ducts. Together, these changes in interstitial $\alpha$ SMA and Coll IV immunoreactivity highlight the rapid and pronounced expansion and remodeling of the outer medullary renal interstitium after obstruction.

\section{Apoptosis}

One of the key features of the early progression of obstructive kidney injury is the onset of epithelial apoptosis, which rapidly impairs tubular function and promotes tubular atrophy. ${ }^{30-35}$ We used TUNEL labeling to assay apoptosis in kidneys obstructed for 1, 2, 3, 6, and 9 days. In sham-operated kidneys at all time points, TUNEL-positive cells were rare and scattered throughout the kidney (Figures $4 \mathrm{a}-\mathrm{c})$ and are indicative of the normal rate of cell turnover within the kidney.

In kidneys obstructed for up to 3 days, the frequency of TUNEL-positive cells increases $v s$ sham kidneys (Figures $4 \mathrm{~d}-\mathrm{f})$. In the inner medulla, TUNEL-positive events occurred in some dilated ducts and were most common at the boundary of the inner and outer medulla (arrowheads, Figure $4 \mathrm{~d}$ ). In the outer medulla, the pericalyceal tissue immediately adjacent to the distended calyceal space demonstrated substantial parenchymal compression with abundant TUNEL-positive cells and cellular debris in both collecting ducts and adjacent loops of Henlé. In other regions of the outer medulla, apoptosis was mild to moderate and occurred largely in the interstitium and AQP2-negative loop of Henle, with collecting duct apoptosis more limited and present in dilated ducts (Figure 4f). TUNEL-positive cells and cellular debris were present in the lumen of some tubules and ducts. In the cortex, TUNEL-positive cells resided in juxtamedullary tubules displaying proximal tubular morphology and in the distal and connecting tubules of the outer cortex (Figure 4e). 
a
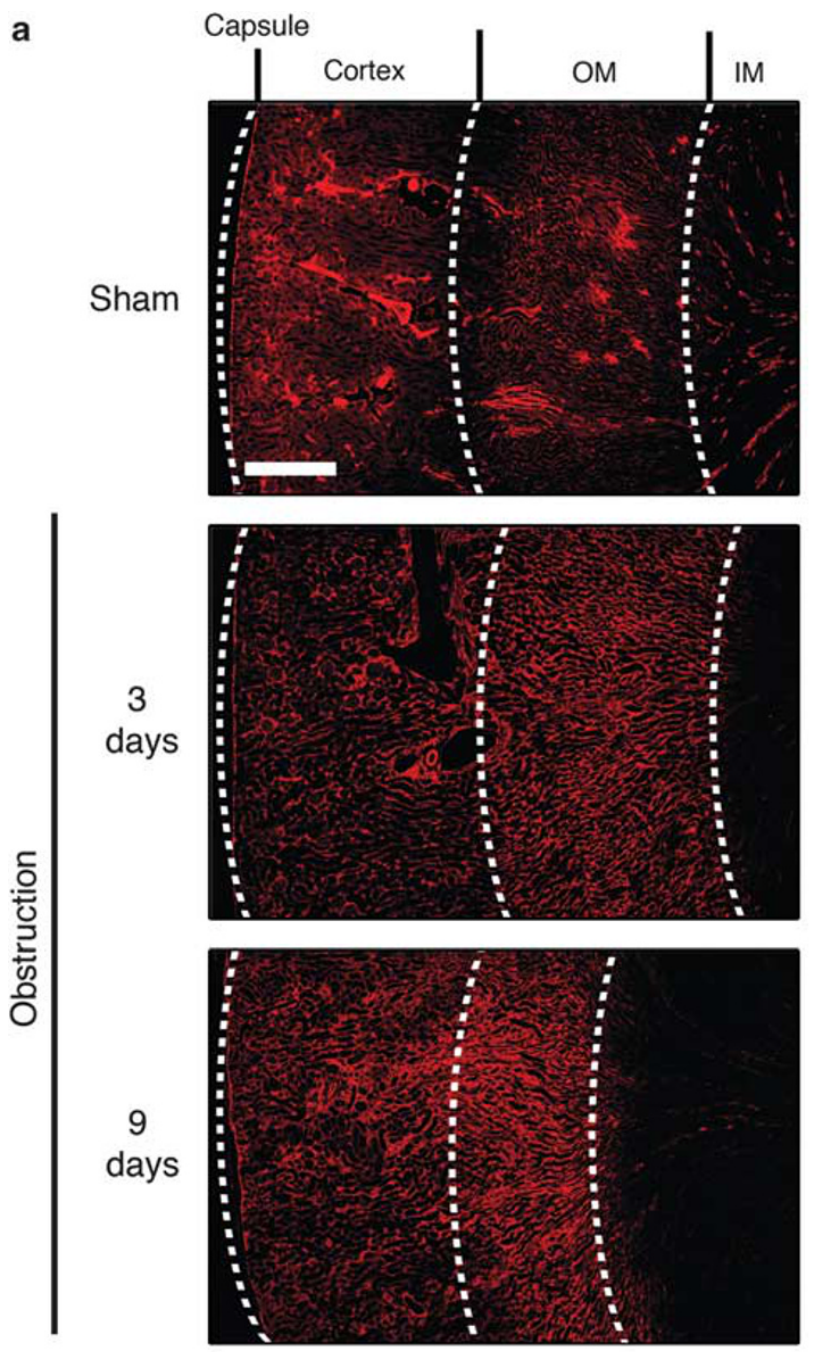

c

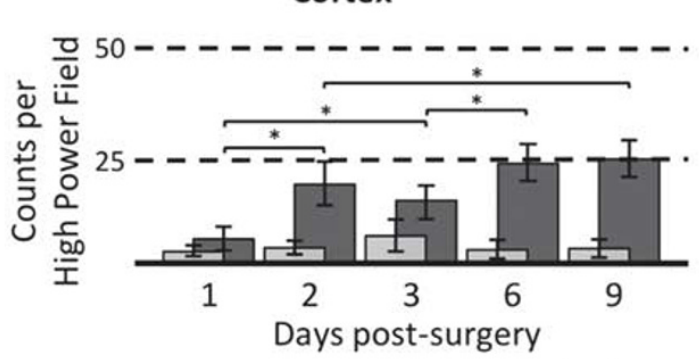

b
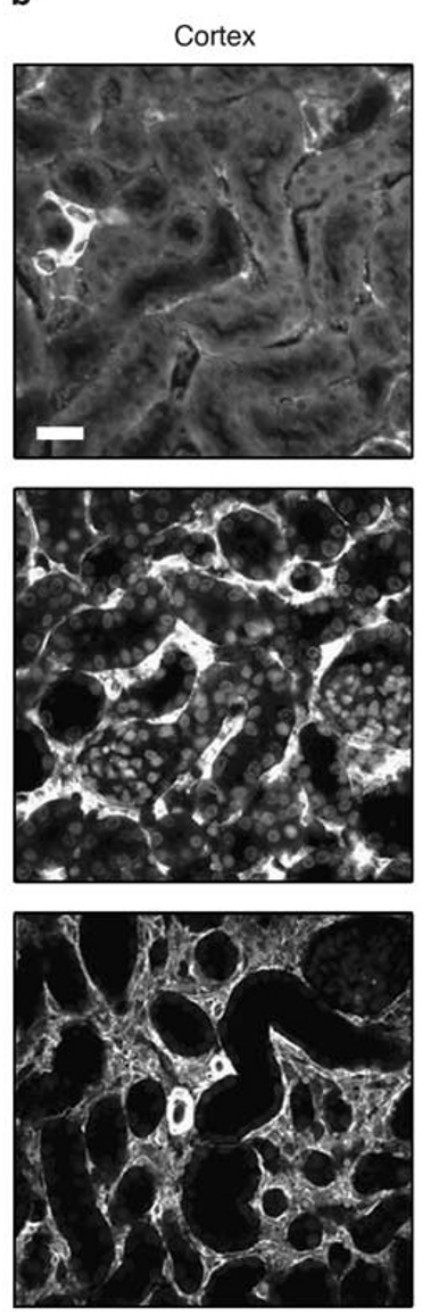

Outer

Medulla
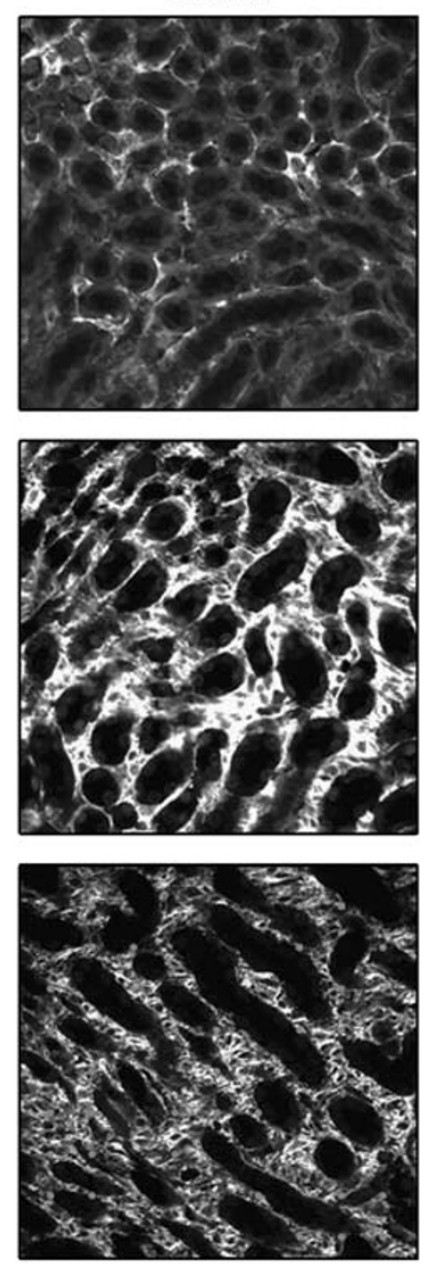

Outer Medulla

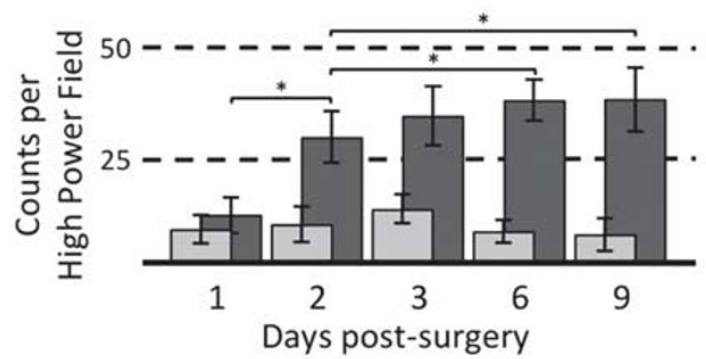

Sham

Obstructed

Figure 2 Accumulation of $\alpha$ SMA-positive myofibroblasts. (a) In sham animals, $\alpha$ SMA (red) is expressed solely in the renal vasculature. Following prolonged obstruction, interstitial $\alpha \mathrm{SMA}$ expression increases progressively, with more dramatic accumulation of interstitial $\alpha \mathrm{SMA}$ in the outer medulla. (b) Representative high-power $(40 \times)$ fields from sham and obstructed animals showing $\alpha$ SMA distribution. (c) When examined via morphometric analysis, $\alpha$ SMA area increased significantly from 1 to 2 days in both outer medulla and cortex, with more gradual $\alpha$ SMA area increase from 2 to 6 days obstruction. In both the outer medulla and cortex, $\alpha$ SMA area reached a plateau from 6 to 9 days. In the obstructed kidneys, all medullary counts were statistically higher than their corresponding cortical counts, ${ }^{*} P<0.05$. Error bars $=$ standard deviation. $N=3$ animals. Scale bars: (a) 1 mm, (b) $25 \mu \mathrm{m}$.

Following 9 days of obstruction, TUNEL-positive apoptotic events were similarly distributed to the 3-day obstructed kidney, but occurred at lower frequency (Figures $4 \mathrm{~g}-\mathrm{i}$ ).
Collecting duct TUNEL-positive events remained largely restricted to the cortical ducts with infrequent medullary events (Figures 4h,i). In the cortex, pyknotic TUNEL-positive 

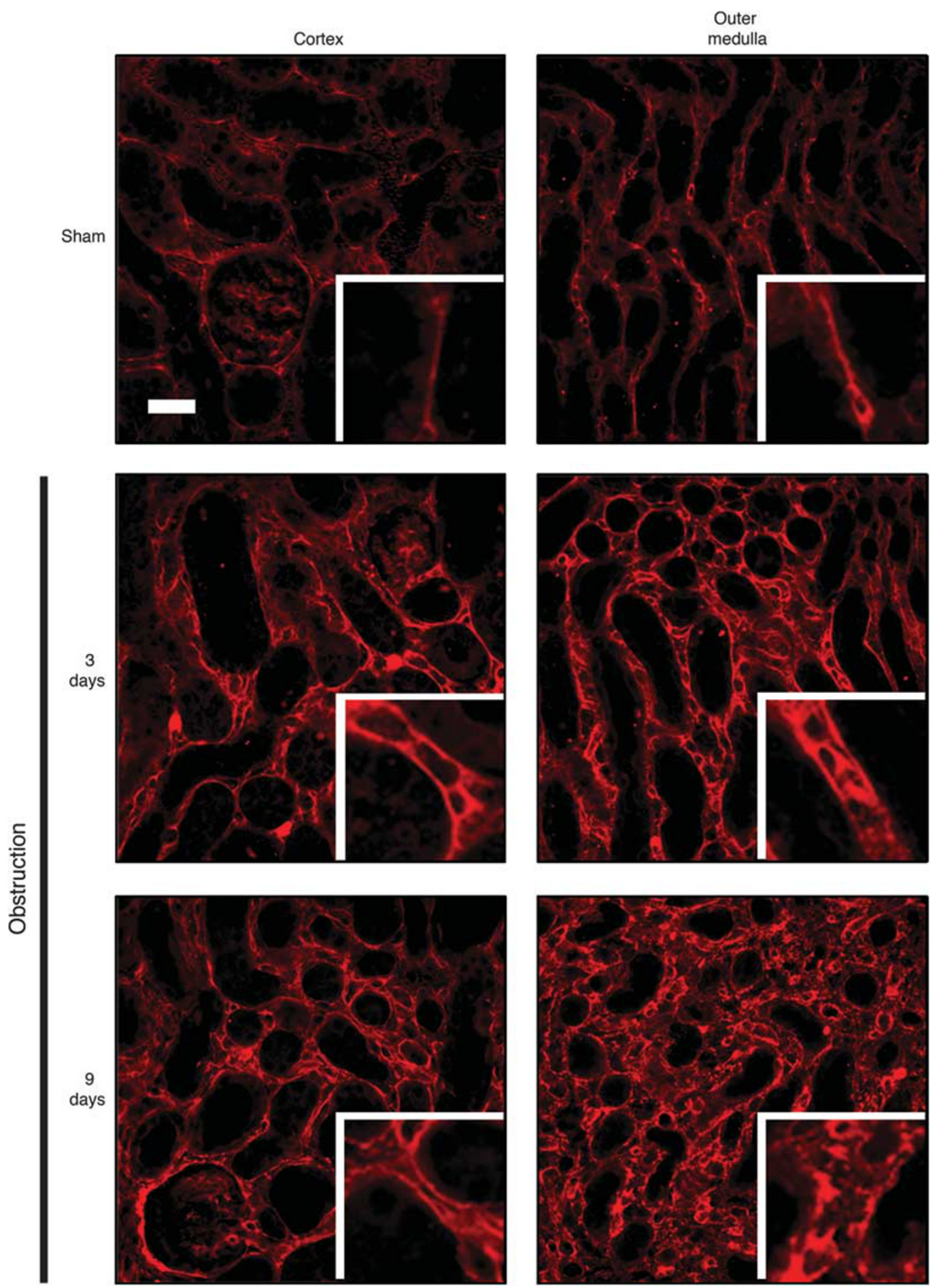

Figure 3 Interstitial collagen IV accumulation. Interstitial Coll IV (red) expression increases progressively with prolonged obstruction, with more dramatic accumulation in the outer medulla than in the cortex. Insets highlight duplication of intratubular basement membranes and expansion of the Coll IV-positive interstitium. Scale bar: $25 \mu \mathrm{m}$.

nuclei were present in segments displaying proximal tubular, interstitial, and to a lesser extent distal tubular morphology (Figure 4h). Additionally, apoptosis was apparent in regions of parenchymal injury at the poles of the renal cortex (Figure 4g, insets). These regions are believed to contain nephrons proximal to the pericalyceal outer medullary regions that were extensively injured early in the obstructive injury and represent the irreparable portions of the kidney described during reversible UUO. ${ }^{29}$ 


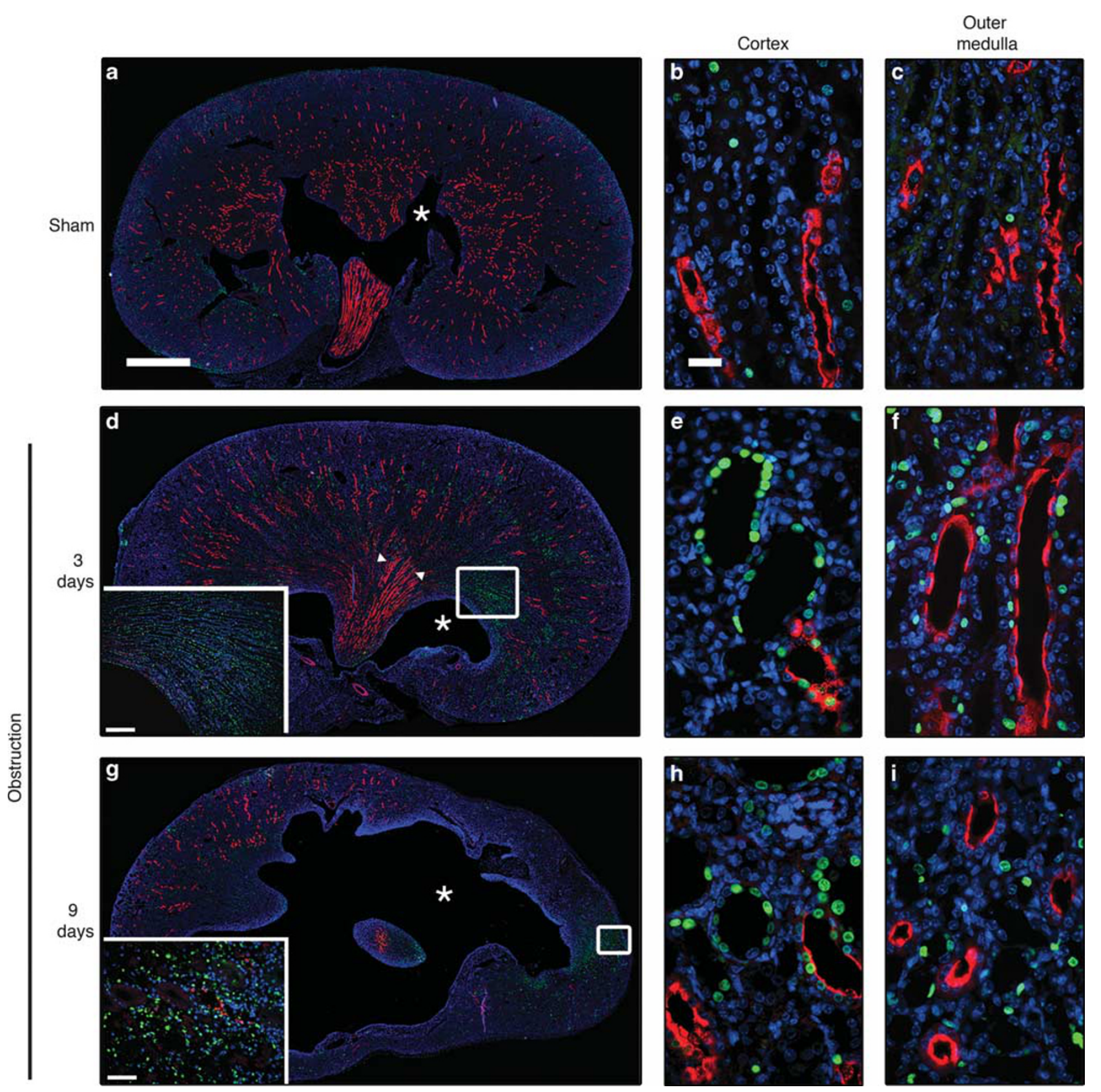

Figure 4 Apoptosis Following UUO. (a) TUNEL-positive cells (green) are rare in the sham-operated kidney and scattered throughout the cortex (b) and medulla (c). Following 3 days of obstruction (d), TUNEL-positive events are increased vs sham. Accumulation of urine in the calyx (asterisk) causes outer medullary compression and substantial apoptosis in the pericalyceal region (box and inset in d). (e) In the cortex, TUNEL-positive cells are most abundant in dilated distal and connecting tubules. (f) Medullary apoptosis occurs predominantly in the interstitium and AQP2-negative loop of Henlé, with fewer events in the AQP2-positive collecting ducts (red). Medullary apoptosis occurs predominantly in the interstitium and AQP2-negative loop of Henlé, with fewer events in the AQP2-positive collecting ducts (red). (g-i) In the 9-day obstructed kidney, apoptosis occurs as in the 3-day kidney, but not as frequently. Atrophied regions of widespread apoptosis and necrosis (box and inset in $\mathbf{g}$ ) are present near the poles of the kidney. Scale bars:

$(\mathbf{a}, \mathbf{d}, \mathbf{g})=1 \mathrm{~mm}$, (inset d) $=200 \mu \mathrm{m}$, (inset $\mathbf{g})=50 \mu \mathrm{m},(\mathbf{b}, \mathbf{c}, \mathbf{e}, \mathbf{f}, \mathbf{h}, \mathbf{i})=25 \mu \mathrm{m}$.

\section{Tubular Dilatation}

Obstruction causes retrograde accumulation of urine and increases in tubular lumen pressure. As early as 1 day after obstruction, dilatation of renal tubules of the outer medulla and cortex could be observed. After 3 days of obstruction, the majority of dilated tubules in the outer medulla were AQP2-positive collecting ducts, whereas adjacent AQP-negative tubules representing segments of the loop of Henlé displayed little or no dilatation. (Figure 5a). Following 9 days of obstruction, all tubular segments of the medulla are dilated 
a
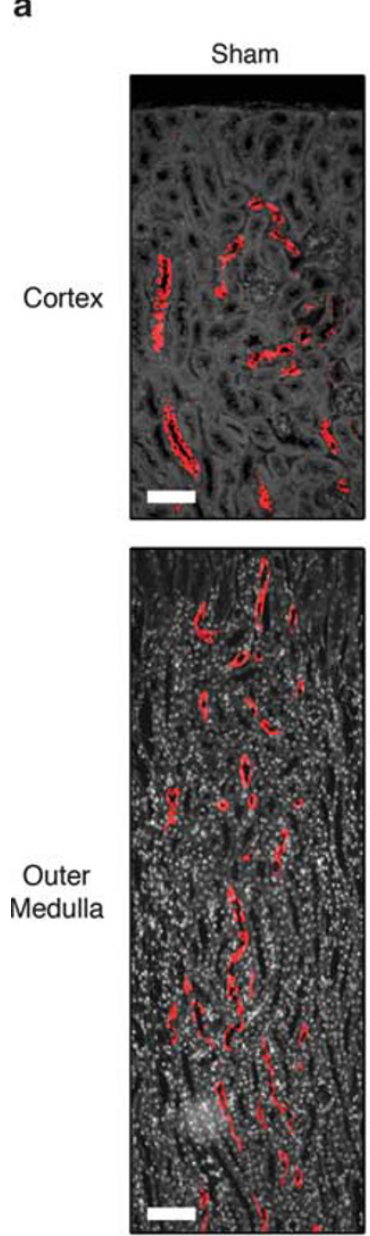

c
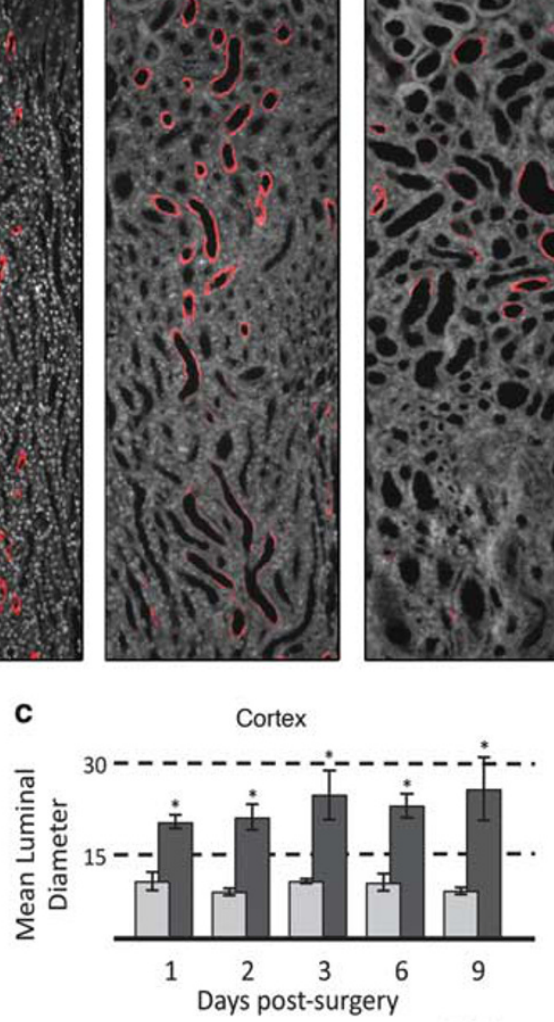

\section{.}

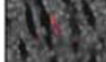

I.t.

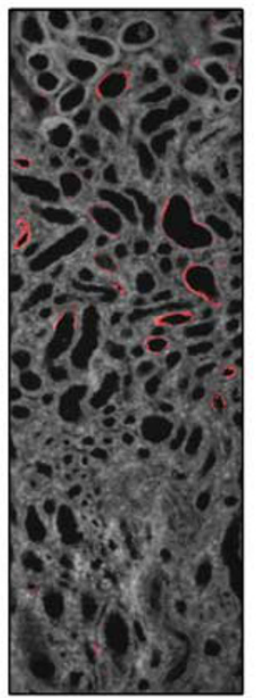

b
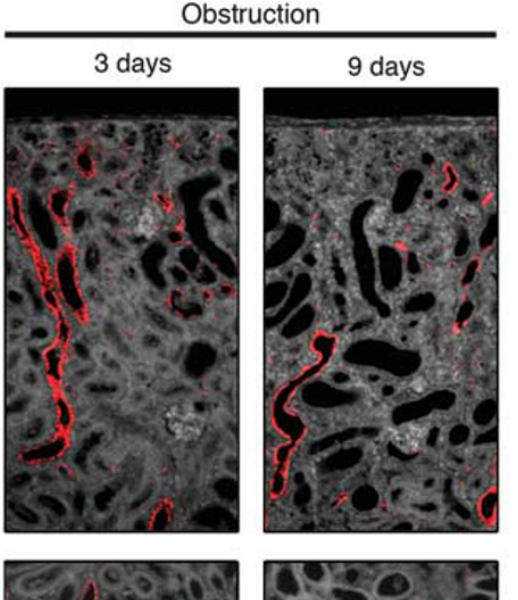
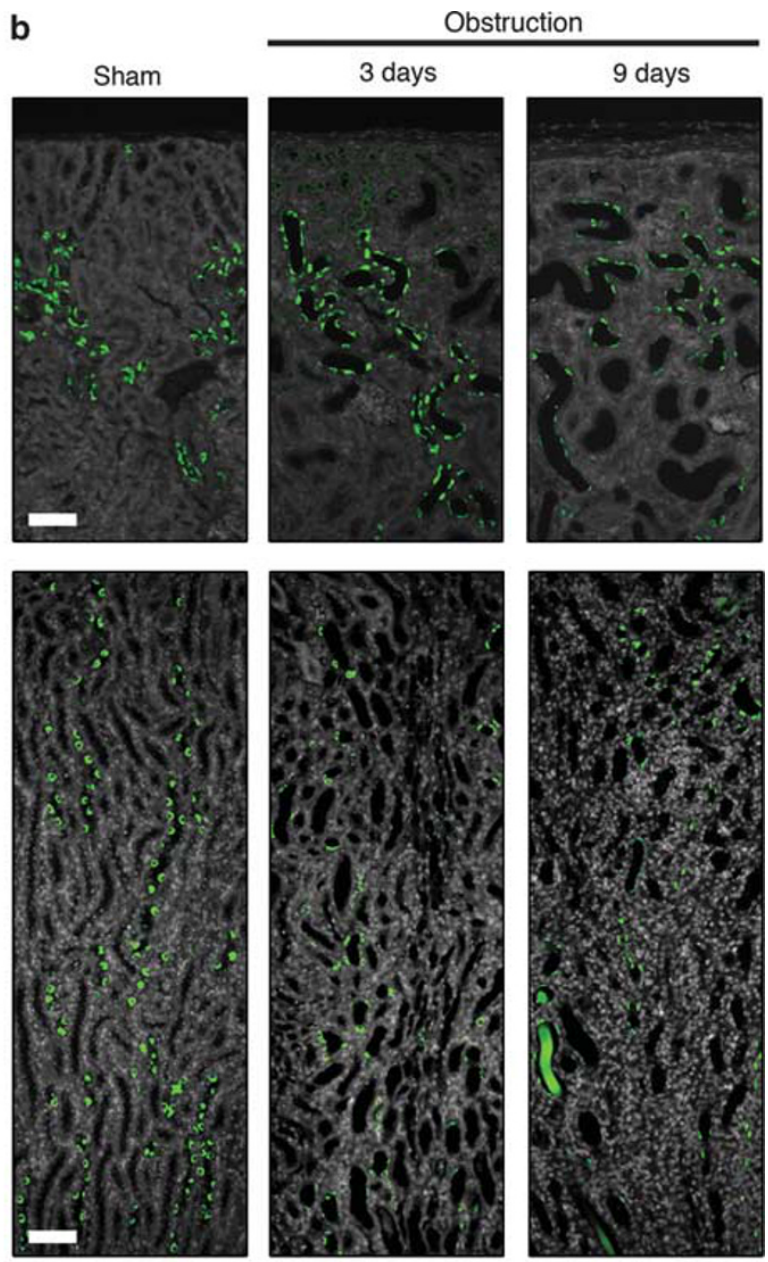

Outer Medulla

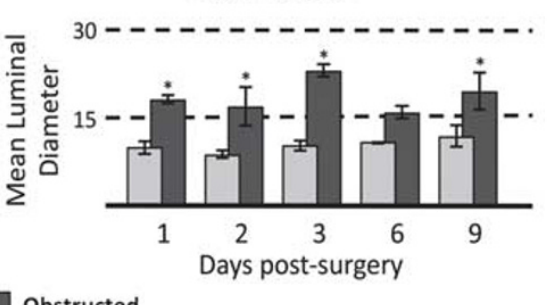

Figure 5 Distal nephron changes following UUO. Tubules of the distal nephron, including (a) AQP2-positive collecting ducts (red) and (b) vATPasepositive distal tubules, connecting tubules, and collecting ducts (green) are dilated at 1-3 days after obstruction, with other nephron segments exhibiting dilatation at 9 days after obstruction. (c) Mean luminal diameter of outer medullary collecting ducts and cortical distal and connecting tubules is increased as early as 1 day post-obstruction and increases progressively with prolonged obstruction. For all tubules and ducts at all time points, with the exception of the 6 day medullary collecting ducts, the mean luminal diameter in the obstructed kidney is significantly larger than the age-matched sham. ${ }^{*} P<0.05$ vs sham. Increased duration of urinary obstruction also results in the progressive loss of (a) AQP2 expression in principal cells (red) and (b) vATPase-expressing intercalated cells (green). AQP2 and vATPase expression levels are most affected in the inner stripe of the outer medulla, with more modest reductions in cortical collecting ducts or distal and connecting tubules, respectively. Scale bars: $100 \mu \mathrm{m}$.

including those of both AQP2-positive collecting duct and AQP2-negative loop of Henlé. Similarly, the majority of dilated tubules in the cortex of the obstructed kidneys consisted of vATPase-positive distal convoluted and connecting tubules (Figure 5b) and, to a lesser degree, cortical collecting ducts. Little or no changes in the morphology or structure of the other cortical nephron segments such as proximal convoluted tubules were evident in early obstruction. At the later time points of 6 and 9 days post-obstruction, dilatation of other tubular segments displaying loop of Henlé and proximal tubular morphology was more apparent (Figure 5). To quantify tubular dilatation, we measured the luminal diameters of 
a
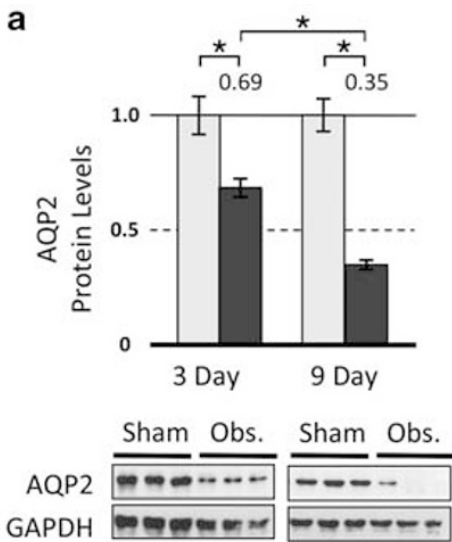

b

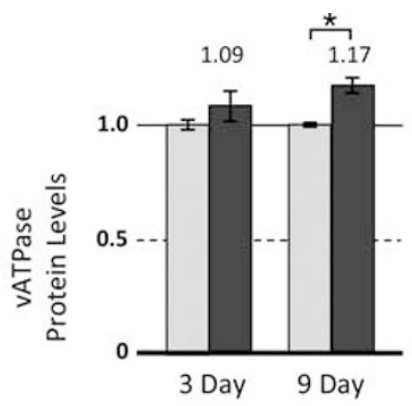

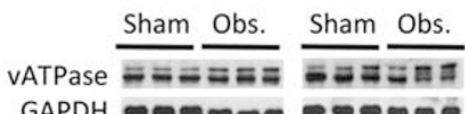

$\square$ Sham $\square$ obstructed

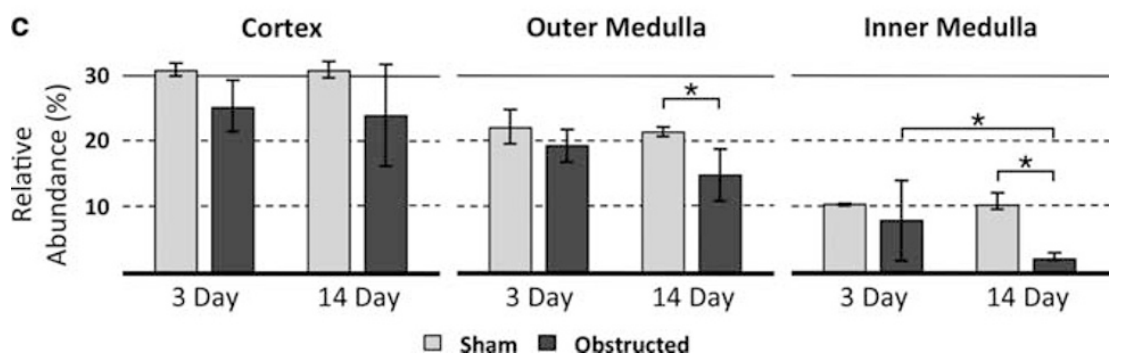

Figure 6 Alteration of principal and intercalated cell markers. Whole kidney protein levels, analyzed by western blot, of principal cell marker AQP2 (a) were reduced in kidneys 3 and 9 days post-obstruction when compared with sham-operated kidneys, whereas intercalated cell marker vATPase (b) increased slightly. As vATPase is also expressed in other tubular segments including distal and connecting tubules, vATPase-positive intercalated cells of the collecting ducts were counted manually (c). These analyses demonstrate reduced numbers of intercalated cells following prolonged obstruction, with the largest decreases in the inner and outer medulla. ${ }^{*} P<0.05$. Error bars = standard deviation. $N=3$ animals.

medullary collecting ducts, and of cortical distal and connecting tubules. Significant dilatation of medullary collecting ducts and cortical distal/connecting tubules occurred as early as 1 day post obstruction (Figure $5 \mathrm{c}$ ). Obstructed collecting ducts and distal/connecting tubules of all time points, with the exception of outer medullary collecting ducts at 6 days obstruction, displayed significantly larger luminal diameters than age-matched shams.

These results suggest that the effects of ureteric obstruction proceed retrograde, affecting the collecting duct and distal convoluted tubule first, then progressing to the loop of Henlé and proximal convoluted tubule.

\section{Collecting Duct Epithelial Changes}

In addition to the changes affecting the interstitial compartment, we have previously shown that ureteric obstruction alters the development and differentiation of the collecting duct epithelium in both human and primate fetal kidneys. The normal collecting duct epithelium is composed of two cell types, with AQP2-positive principal cells constituting the majority of collecting duct cells and vATPase-positive intercalated cells comprising the remainder. In the postnatal murine UUO model, there was a progressive loss of AQP2 immunoreactivity in principal cells, which closely replicates the findings in obstructed fetal human and primate kidneys
(Figure 5a). In the murine kidney, the most significant depletion of AQP2-positive principal cells occurred within the inner stripe of the outer medulla, and to a lesser extent in the inner medulla, whereas cortical collecting ducts exhibited relatively minor changes. Similarly, ureteric obstruction resulted in the loss of vATPase-expressing intercalated cells (Figure 5b). Following 9 days of obstruction, the protein levels of AQP2 in kidney lysates decreased dramatically as determined by western blot analysis (Figure 6a), whereas whole kidney vATPase protein levels slightly increased (Figure 6b). As vATPase-positive intercalated cells appear reduced following obstruction, especially in the outer medulla (Figure 5b), it is likely this increase in vATPase protein levels reflects altered expression in the other vATPaseexpressing nephron segments including the distal convoluted tubule and ascending loop of Henlé.

To better assess the effects of obstruction on intercalated cell numbers, we counted vATPase-positive cells in representative fields of the cortex, outer medulla, and inner medulla. In sham animals, intercalated cells represented $31 \%, 22 \%$, and $11 \%$ of the cortical, outer medullary, and inner medullary collecting duct epithelium, respectively (Figure 6c). Following 3 days obstruction, intercalated cell abundance shows no significant decrease in any region. However, our previous studies in human and primate fetal 
a
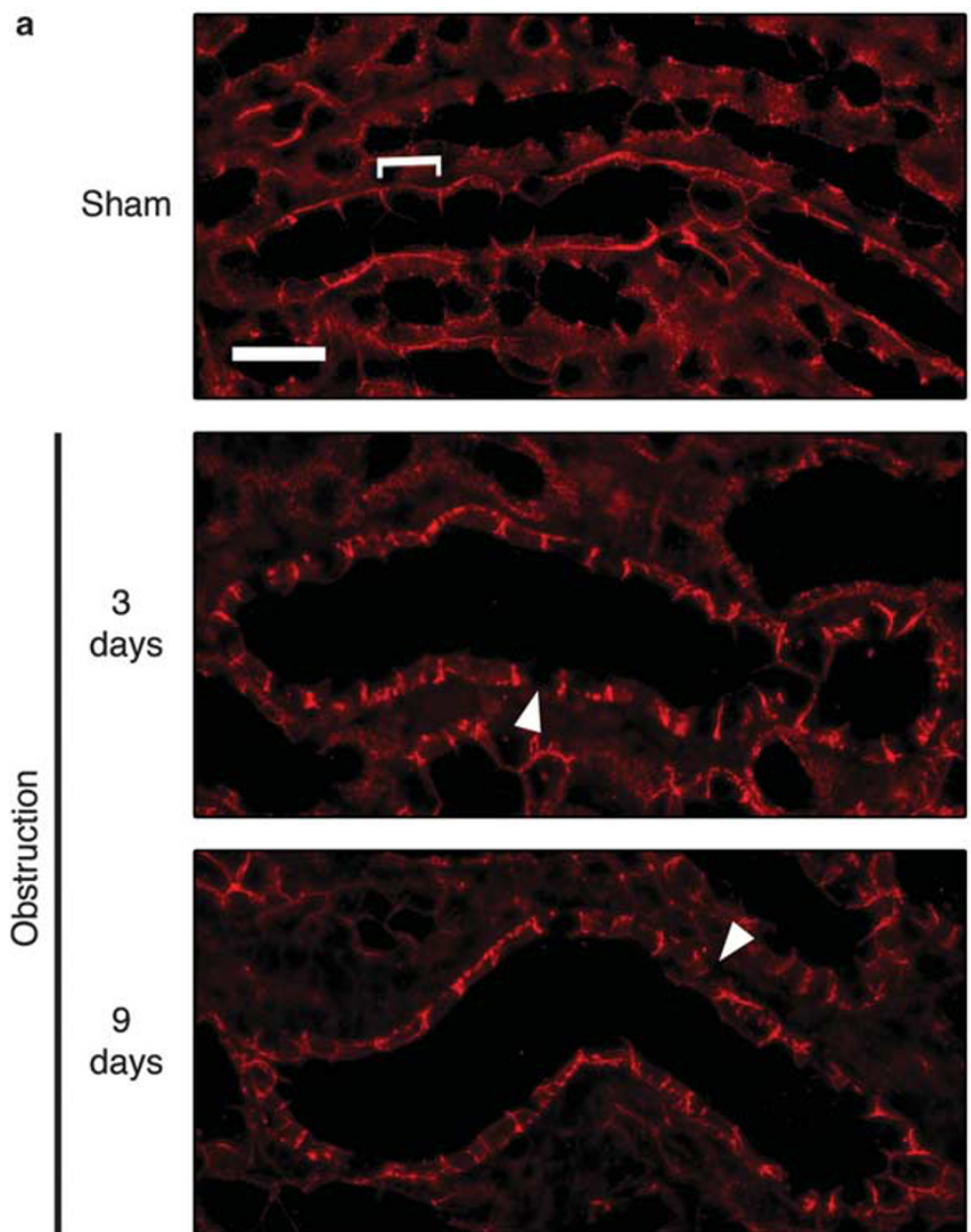

b
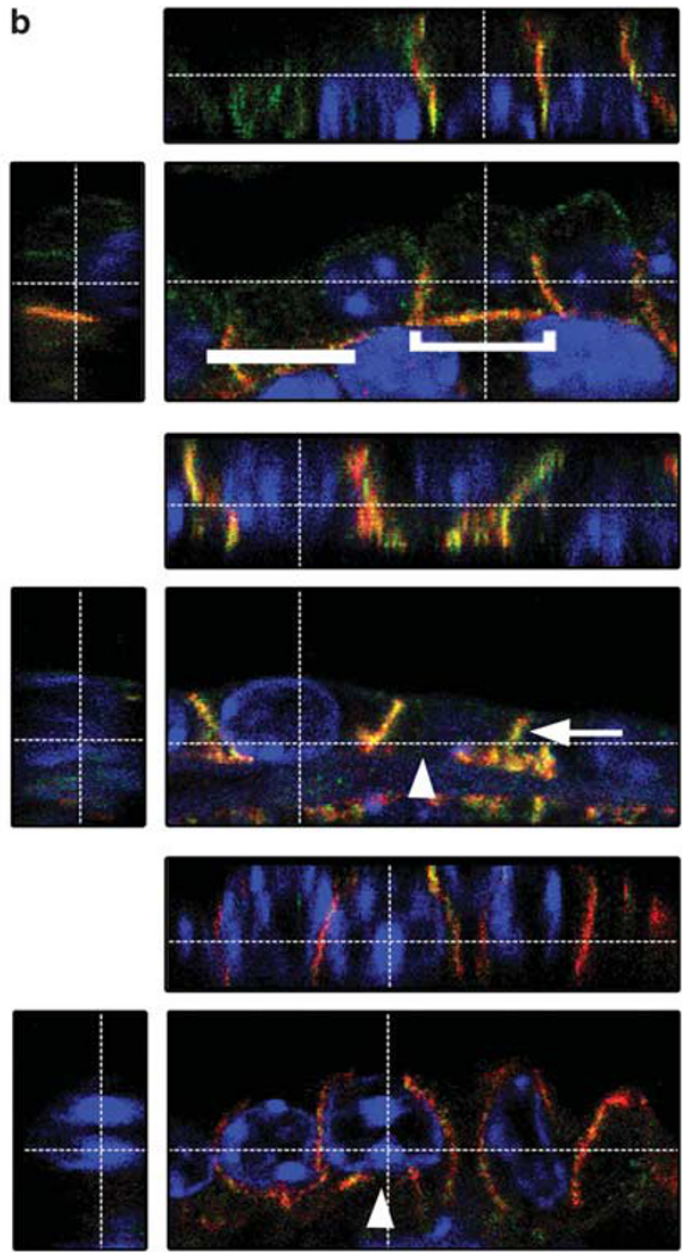

Figure 7 Disruption of ECad and $\beta$ Cat localization. (a) ECad expression in the outer medullary collecting duct and (b) confocal imaging of ECad and $\beta$ Cat in the collecting duct epithelial cells. ECad-expressing collecting duct cells display regular epithelial patterning (bracket) in sham-operated kidneys, with colocalization of ECad and $\beta$ Cat at the basolateral membrane. Following 3 and 9 days of obstruction, ECad and $\beta$ Cat expression levels are lost at the basal membrane (arrowhead), whereas expression and colocalization at the lateral membranes are more diffuse (arrow). At 9 days of obstruction, collecting duct cells have lost their cuboidal morphology, display decreased ECad and $\beta$ Cat colocalization, and become amorphous. Scale bars: (a) $=25 \mu \mathrm{m},(\mathbf{b})=10 \mu \mathrm{m}$.

kidneys have described more substantial decreases in medullary intercalated cell abundance (32-67\% decrease at near term gestation) following prolonged, chronic obstruction. ${ }^{1}$ To replicate these conditions as closely as possible in the murine model of UUO, we counted intercalated cells in kidneys obstructed for 14 days. This prolonged obstruction resulted in a more significant reduction of intercalated cell numbers in the outer and inner medulla (decreases of $31 \%$ and $95 \%$, respectively) similar to that in chronic human and primate fetal kidney obstruction. These results compare favorably with our previous observations in the fetal human and primate kidney and confirm that the loss of AQP2 and vATPase expression in principal and intercalated cells were common features of both fetal and postnatal urinary tract obstruction.

The disruption of epithelial adherens junctions is another well-characterized hallmark of epithelial injury. The association of ECad and its binding partner $\beta$ Cat is essential for the formation of epithelial adherens junction complexes, whereas the disassociation of these complexes promotes the loss of a differentiated epithelial phenotype. Furthermore, free cytoplasmic $\beta$ Cat can be translocated to the nucleus where it acts as a transcriptional activator of many genes involved in promoting repair or fibrosis. As in the obstructed fetal primate kidney, obstruction in the mouse altered the normal cuboidal morphology of collecting duct cells producing an irregularly patterned, amorphous epithelium (Figure 7a). Confocal microscopy demonstrated that ECad and its binding partner $\beta$ Cat are tightly colocalized at the basolateral membrane of the sham collecting duct epithelium (Figure 7b). After 3 days of obstruction, lateral membrane colocalization of ECad and $\beta$ Cat was more diffuse, whereas basal membrane expression and colocalization is lost. By 9 days of obstruction, medullary collecting duct epithelial 
cells have lost their normal cuboidal structure, displaying irregular epithelial morphology with diffuse and irregular lateral membrane ECad localization (Figure 7b).

These results indicate that obstruction alters the structure and cellular composition of the collecting duct epithelium. The loss of the functional transporters AQP2 and vATPase in principal cells and intercalated cells, respectively, in conjunction with the disruption of ECad localization, suggest that obstruction causes cellular dedifferentiation and promotes mesenchymal transformation of the collecting duct epithelium.

\section{DISCUSSION}

Much of our understanding of the pathophysiology of kidney injury with urinary tract obstruction comes from work performed in postnatal mouse models focusing on fibrosis as the primary outcome. We have previously characterized several key steps in the pathogenesis of fetal obstructive nephropathy in both human and primate kidneys and highlighted an important role for collecting duct injury and remodeling. In this report, we used the murine postnatal UUO model to characterize distal nephron injury, including the collecting ducts, connecting tubules, and distal tubules.

Obstruction initiates important early events. In particular, gross distension of the ureter and renal calyces compresses medullary and cortical tissue. Notably, the outer medullary region immediately adjacent to the calyx exhibits substantial compression resulting in widespread apoptosis and tubular atrophy as early as 3 days post-obstruction. The nephron segments of these medullary regions correspond to the nephrons of the renal cortex where widespread and irreversible cortical atrophy and apoptosis occur. ${ }^{29}$ In addition, dilatation of the tubules occurs in a retrograde manner and first impacts the collecting duct, connecting tubule, and distal tubule segments. These observations emphasize the magnitude of the physical stresses, the rapid onset, and the retrograde progression of kidney injury with urinary tract obstruction.

The interstitial response to urinary tract obstruction is also an important contributor to progressive injury in both fetal and postnatal models. It is characterized by interstitial expansion, alteration of collagen deposition, and the recruitment or production of myofibroblasts. In the fetal human and primate, obstruction is associated with reorganization and thickening of Coll IV expression in the tubular basement membranes, and with the formation of $\alpha$ SMA-positive peritubular collars. ${ }^{1,3}$ In the UUO model, Coll IV and $\alpha$ SMApositive myofibroblasts also accumulate within the interstitium, but are more widely distributed throughout the interstitium. This difference likely reflects the more rapid onset and more severe injury caused by complete obstruction with ureteric ligation, compared with the more gradual progression invoked by partial or incomplete obstruction, as in fetal human congenital obstruction or in the fetal primate model of obstruction.

The role of interstitial Coll IV and $\alpha$ SMA accumulation during early obstruction remains unclear. Coll IV remodeling and deposition may reflect disruption of the basement membrane as part of the process of phenotypic transition or epithelial mesenchymal transition, or as a response to injury. Similarly, the significance of the dramatic recruitment of aSMA-positive cells into the renal interstitium as early as 2 days post-obstruction is unknown. It is possible this early activation of putative $\alpha$ SMA-positive myofibroblasts serves as an antecedent to the fibrosis seen with prolonged injury. However, it is likely that these cells also have a role in the early response to obstructive injury, and may reflect part of the normal acute repair response. As with the deposition of interstitial collagen, this recruitment of $\alpha \mathrm{SMA}$-expressing cells may also serve to decrease tubular compliance and mitigate subsequent injury.

As observed during obstruction in the fetal human and primate kidney, murine UUO results in the remodeling and dedifferentiation of the collecting duct and distal nephron epithelium. Notably, the number of AQP2-positive principal cells and vATPase-positive intercalated cells are reduced. Furthermore, as seen in the obstructed primate kidney, ${ }^{3}$ obstruction causes morphological changes in the collecting duct epithelia, including disruption and dislocation of ECad expression. ECad remodeling is a hallmark feature of epithelial injury that generally precedes cellular depolarization, loss of the associated tight junctions, and dedifferentiation of epithelial cells. ${ }^{36}$ These steps are considered key in the acquisition of a less differentiated or mesenchymal phenotype that may be necessary to allow for sufficient epithelial plasticity to permit appropriate epithelial repair.

Obstruction of the postnatal mouse kidney results in distal nephron injury similar to that which we have previously described in the fetal human and primate. Specifically, significant early remodeling of the medullary and cortical collecting duct occurs following obstruction, but has been largely underrepresented in the published work in the mouse UUO model. However, these results complement recently reported findings following prolonged obstruction including increased parenchymal compression, distal nephron dilatation, and interstitial SMA accumulation. ${ }^{37}$

When compared with fetal urinary tract obstruction, these findings underscore collecting duct epithelial injury and remodeling as common and conserved features of obstructive injury. They highlight early injury-mediated activation of the distal nephron epithelia, and the likelihood that the magnitude and nature of this activation modulates subsequent injury and repair.

\section{ACKNOWLEDGEMENTS}

We thank the Child and Family Research Institute's Histology and Imaging Core for use and maintenance of the imaging suite. This work was supported in part by the Transplant Research Foundation and by the British Columbia Provincial Renal Agency.

\section{DISCLOSURE/CONFLICT OF INTEREST}

The authors declare no conflict of interest. 
1. Hiatt $\mathrm{M}$, Ivanova $\mathrm{L}$, Toran $\mathrm{N}$, et al. Remodeling of the fetal collecting duct epithelium. Am J Pathol 2010;176:630-637.

2. Trnka $P$, Hiatt $M$, Ivanova $L$, et al. Phenotypic Transition of the Collecting Duct Epithelium in Congenital Urinary Tract Obstruction. J Biomed Biotechnol 2010;2010:1-10.

3. Butt MJ, Tarantal AF, Jimenez DF, et al. Collecting duct epithelialmesenchymal transition in fetal urinary tract obstruction. Kidney Int 2007;72:936-944.

4. Matsell DG, Mok A, Tarantal AF. Altered primate glomerular development due to in utero urinary tract obstruction. Kidney Int 2002;61:1263-1269.

5. Chevalier RL. Obstructive nephropathy: towards biomarker discovery and gene therapy. Nat Clin Pract Nephrol 2006;2:157-168.

6. Chevalier RL, Forbes MS, Thornhill BA. Ureteral obstruction as a mode of renal interstitial fibrosis and obstructive nephropathy. Kidney Int 2009:75:1145-1152.

7. Chevalier RL, Thornhill BA, Forbes MS, et al. Mechanisms of renal injury and progression of renal disease in congenital obstructive nephropathy. Pediatr Nephrol 2010;25:687-697.

8. Chevalier RL. Pathogenesis of renal injury in obstructive uropathy. Curr Opin Pediatr 2006;18:153-160.

9. Oda T, Jung YO, Kim HS, et al. PAl-1 deficiency attenuates the fibrogenic response to ureteral obstruction. Kidney Int 2001;60: 587-596.

10. Zhang G, Kim H, Cai X, et al. Urokinase receptor deficiency accelerates renal fibrosis in obstructive nephropathy. J Am Soc Nephro 2003;14:1254-1271.

11. Lopez-Guisa JM, Cai X, Collins SJ, et al. Mannose receptor 2 attenuates renal fibrosis. J Am Soc Nephrol 2012;23:236-251.

12. Fujiu K, Manabe I, Nagai R. Renal collecting duct epithelial cells regulate inflammation in tubulointerstitial damage in mice. J Clin Invest 2011;121:3425-3441.

13. Josephson S, Robertson B, Claesson G, et al. Experimental obstructive hydronephrosis in newborn rats. I. Surgical technique and long-term morphologic effects. Invest Urol 1980;17:478-483.

14. Josephson S, Ericson AC, Sjöquist M. Experimental obstructive hydronephrosis in newborn rats. VI. Long-term effects on glomerular filtration and distribution. J Urol 1985;134:391-395.

15. Stenberg A, Olsen L, Josephson $S$, et al. Partial ureteric obstruction in weanling rats. I. Long-term effects on the renal morphology. Scand J Urol Nephrol 1985;19:139-146.

16. Chung KH, Chevalier RL. Arrested development of the neonatal kidney following chronic ureteral obstruction. J Urol 1996;155:1139-1144.

17. Fern RJ, Yesko CM, Thornhill BA, et al. Reduced angiotensinogen expression attenuates renal interstitial fibrosis in obstructive nephropathy in mice. J Clin Invest 1999:103:39-46.

18. Martinez-Maldonado M, Delafontaine $P$, Anwar A, et al. Aortic and renal regulation of the renin-angiotensin system in interrenal aortic coarctation. Trans Assoc Am Physicians 1993;106:120-127.

19. Pimentel JL, Martinez-Maldonado M, Wilcox JN, et al. Regulation of renin-angiotensin system in unilateral ureteral obstruction. Kidney Int 1993:44:390-400
20. Gerth JH, Kriegsmann J, Trinh TT, et al. Induction of p27KIP1 after unilateral ureteral obstruction is independent of angiotensin II. Kidney Int 2002;61:68-79.

21. Grande MT, López-Novoa JM. Fibroblast activation and myofibroblast generation in obstructive nephropathy. Nat Rev Nephrol 2009;5: 319-328.

22. Eddy AA. Molecular basis of renal fibrosis. Pediatr Nephrol 2000; 15:290-301.

23. Strutz F, Zeisberg M. Renal fibroblasts and myofibroblasts in chronic kidney disease. J Am Soc Nephrol 2006;17:2992-2998.

24. Zeisberg M, Neilson EG. Mechanisms of tubulointerstitial fibrosis. J Am Soc Nephrol 2010;21:1819-1834.

25. Kim H, Oda T, López-Guisa J, et al. TIMP-1 deficiency does not attenuate interstitial fibrosis in obstructive nephropathy. J Am Soc Nephrol 2001;12:736-748.

26. Kuwabara $\mathrm{N}$, Tamada $\mathrm{S}$, Iwai $\mathrm{T}$, et al. Attenuation of renal fibrosis by curcumin in rat obstructive nephropathy. Urology 2006;67: 440-446.

27. Miyajima A, Chen J, Lawrence C, et al. Antibody to transforming growth factor-beta ameliorates tubular apoptosis in unilateral ureteral obstruction. Kidney Int 2000;58:2301-2313.

28. Sharma AK, Mauer SM, Kim Y, et al. Altered expression of matrix metalloproteinase-2, TIMP, and TIMP-2 in obstructive nephropathy. J Lab Clin Med 1995;125:754-761.

29. Cochrane AL, Kett MM, Samuel CS, et al. Renal structural and functional repair in a mouse model of reversal of ureteral obstruction. J Am Soc Nephrol 2005;16:3623-3630.

30. Truong LD, Petrusevska G, Yang G, et al. Cell apoptosis and proliferation in experimental chronic obstructive uropathy. Kidney Int 1996;50:200-207.

31. Gobe GC, Axelsen RA. Genesis of renal tubular atrophy in experimental hydronephrosis in the rat. Role of apoptosis. Lab Invest 1987;56: 273-281.

32. Miyajima A, Chen J, Poppas DP, et al. Role of nitric oxide in renal tubular apoptosis of unilateral ureteral obstruction. Kidney Int 2001; 59:1290-1303.

33. Gonçalves RG, Gabrich L, Rosário A, et al. The role of purinergic P2 $\times 7$ receptors in the inflammation and fibrosis of unilateral ureteral obstruction in mice. Kidney Int 2006;70:1599-1606.

34. Cohen T, Loutochin O, Amin M.C, et al. PAX2 is reactivated in urinary tract obstruction and partially protects collecting duct cells from programmed cell death. Am J Physiol Renal Physiol 2007;292: F1267-F1273.

35. Thornhill BA, Forbes MS, Marcinko ES, et al. Glomerulotubula disconnection in neonatal mice after relief of partial ureteral obstruction. Kidney Int 2007:72:1103-1112.

36. Ishibe S, Cantley LG. Epithelial-mesenchymal-epithelial cycling in kidney repair. Curr Opin Nephrol Hypertens 2008;17:379-385.

37. Forbes MS, Thornhill BA, Minor JJ, et al. Fight-or-flight: murine unilateral ureteral obstruction causes extensive proximal tubular degeneration, collecting duct dilatation, and minimal fibrosis. Am J Physsiol Renal Physiol 2012;303:F120-F129. 